\title{
Early diagnosis of Turner Syndrome Phenotype in a Newborn With Mild Symptoms and Concomitant Neonatal Jaundice
}

\section{ISSN: 2576-9200}

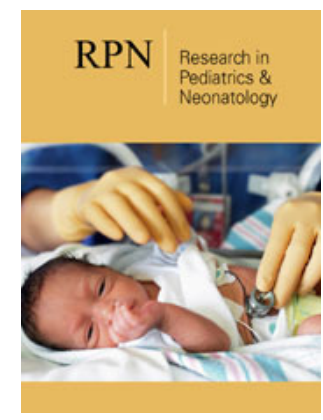

*Corresponding author: Halyna Bulak, Associate Professor, Department of Pediatrics, Lviv City Children Hospital, Danylo Halytsky Lviv National Medical University, Ukraine

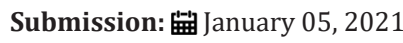

Published: 漈February 1, 2021

Volume 5 - Issue 3

How to cite this article: Halyna Bulak , Andriy Zanevych. Early diagnosis of Turner Syndrome Phenotype in a Newborn With Mild Symptoms and Concomitant Neonatal Jaundice. Research in Pediatrics \& Neonatology. 5(3). RPN. 000612. 2021. DOI: $10.31031 /$ RPN.2021.05.000612

Copyright $@$ Halyna Bulak. This article is distributed under the terms of the Creative Commons Attribution 4.0 International License, which permits unrestricted use and redistribution provided that the original author and source are credited.

\section{Halyna Bulak ${ }^{1 *}$ and Andriy Zanevych ${ }^{2}$}

${ }^{1}$ Associate Professor,Department of Pediatrics, Lviv City Children Hospital, Danylo Halytsky Lviv National Medical University, Ukraine

${ }^{2}$ Danylo Halytsky Lviv National Medical University, Ukraine

\begin{abstract}
Turner syndrome is a rare genetic disorder that occurs only in girls. It can cause problems ranging from short height to heart defects. Sometimes, the symptoms are so mild that it goes undiagnosed until a female is a teen or young adult. That's why, early diagnosis plays a crucial role in further child's development. A lot of health problems affecting girls with Turner syndrome can be managed with treatment. This article represents a clinical case of Turner syndrome phenotype diagnosed on its early stage.
\end{abstract}

Keywords: Turner syndrome;Chromosome anomaly;Neonatal jaundice;Heart defects;Karyotype

Abbreviations: TS: Turner Syndrome; AP: Arterial Pressure; ARVI: Acute Respiratory Viral Infection; TSH: Thyroid: Stimulating Hormone; FT4: Free Thyroxine

\section{Introduction}

Turner syndrome (also known as $45 \mathrm{X}$ or $45 \mathrm{X} 0$ ) is a genetic condition in which a female is partly or completely missing an $\mathrm{X}$ chromosome. This pathologyoccurs in one out of every 2,500 to 3,000 live female births [1]. Turner syndrome in newborns is characterized by the following symptoms: wide or weblike neck, swelling of the hands and feet, broad chest with widely spaced nipples, low hairline at the back of the head, heart defects. Therefore, early detection and diagnosis of Turner syndrome in newborns provides a favorable prognosis of the child's further development.

\section{Case Presentation}

Described below is a medical case of a child suffering from Turner syndrome. At the age of two days the newborn girl was hospitalized to Department of Anesthesia and Intensive Care for Neonates of Lviv City Children hospital having complaints of severe yellowing of the skin and mucous membranes. According to life history, the baby was born from the first pregnancy, the first delivery. During pregnancy, the mother had three cases of acute respiratory viral infection and there was a threat of miscarriage. Newborn condition was moderate severity, body weight: 3300 grams, length: $54 \mathrm{~cm}$, 38 weeks of gestation, Apgar score at birth reached $8 / 8$ points. The following actions were pursued in the delivery room: treatment of upper respiratory tract, aspiration of stomach contents. The state of dynamics was deteriorating due to bilirubin level increase (the general bilirubin level: 299,1 umol/l) and yellowing of the skin. On the second day of life the patient was transferred by neonatal transport crew to the Department of Anesthesia and Intensive Care for Neonates Lviv City Children hospital for further treatment and examination.

At the time of the medical check: up in Lviv City Children hospital, the baby's general condition was close to moderate. Vital signs: a body temperature of $36,7^{\circ} \mathrm{C}$, body weight: 3090 grams, respiratory rate of 50 breaths/minute, heart rate of 150 beats/minute, arterial pressure on the leg: $77 / 24 \mathrm{mmHg}$, AP on the arm: 65/44mmHg, Sp02: $96 \%$. The baby responded to the examination with motor restlessness. The patient's state of consciousness was preserved. Physiological reflexes of the newborn were not stable. There was no need for oxygenation therapy. Superior fontanelle dimensions were $1,5 \times 1,5 \mathrm{~cm}$. The pupillary 
response was retained $\mathrm{D}=\mathrm{S}$. The cutaneous integument was yellow. Visible mucous membranes were clean and wet. Nose breathing was free. Pulmonary auscultation revealed vesicular respiration. Cardiovascular auscultation revealed rhythmic, sonorous heart tones. No abdominal distension was detected, abdomen was soft, painless, available for palpation in all sections. Peristalsis was preserved. Umbilical cord stump was treated with antiseptic. Liver palpating up to $1,5 \mathrm{~cm}$ below the costal arch. The urogenital system was developed in female type, large labia covered small labia. Spleen did not protrude from the edge of the costal arch. Urinary output was autonomous, unstimulated. There was no defecation at the time of the examination. No changes were detected in musculoskeletal system. Given the exposure of the baby to ARVI during pregnancy, prenatal infection was not out of the question. Among laboratory tests: blood test, coagulogram, urinalysis without pathological changes. Biochemistry revealed increased level of bilirubin: 278,9umol/l. Neurosonography demonstrated absence of pathological modifications. Ultrasound of the abdominal organs was normal. A child neurologist detected no changes in nervous system. Treatment with phototherapy, B vitamins and feeding high: adapted mixtures was recommended.

At the age of three days the baby was transferred to newborn pathology department with diagnosis neonatal jaundice. The severe yellowing of the skin and mucous membranes, a short and wide neck and an explicit occipital fold were defined during examination. The recommendation was to continue the ongoing treatment, a number of examinations were scheduled too. General blood and urine tests did not detect any pathological changes. The genetician's consultation revealed that the female proband had Turner syndrome phenotype. It was necessary to carry out cytogenetic examination and urinalysis test to verify the diagnosis. The analysis of the TSH level, phenylalanine and 17: oxyprogesterone in blood was recommended. The urinanalysis for urinalysis proved normal. Measurement of thyroid gland hormone levels and thyroid: stimulating hormone (TSH) were performed, results: TSH: 2,83mm0/l, FT4: $18,4 \mathrm{nmol} / \mathrm{l}$, when normal values are TSH: 0,177: 4,05mm0/l, FT4 : 11,5: 23,0nmol/l. Phenylalanine level in blood : $0,7 \mathrm{mg} / \mathrm{dl}$ while the norm is $0,1: 3,0 \mathrm{mg} / \mathrm{dl} .17$ : oxyprogesterone level was $7,9 \mathrm{ng} / \mathrm{ml}$ with the norm up to $20 \mathrm{ng} / \mathrm{ml}$. The karyotyping was performed according to FISH technique and it revealed 45X: chromosomes. An echocardiography was performed, the following results were yielded: patent foramen ovale: $3 \mathrm{~mm}$ and bicuspid aortic valve without dysfunction. An ultrasound of the hip joints was also performed according to the Graph's method: the first joint type (mature joint) was found both on the right and on the left. Based on all clinical data, physical examination as well as laboratory and instrumental examination, karyotyping, the final diagnosis for this patient was neonatal jaundice, Turner syndrome phenotype.

Prescribed treatment, that included feeding high: adapted mixture, phototherapy, vitamins $\mathrm{B}_{1}$ (thiamine) and $\mathrm{B}_{6}$ (pyridoxine) in dosage of $0,3 \mathrm{mg}$ once a day intravenously, balanced feeding produced good results. The jaundice reduced. The biochemistry revealed a gradual decline in the general bilirubin level from 299,7 umol/l to 181,7 umol/l. At the age of 21 day, the child was discharged from the newborn pathology department and returned home in good condition. Parents received some important recommendations regarding breastfeeding and further pediatric, neurological and cardiosurgical follow: up. It was recommended to take one drop of vitamin $\mathrm{D}_{3}$ (cholecalciferol) once per day.

\section{Discussion}

The Turner syndrome signs such as a short and webbed neck, cardiac defects in this case can be caused by the partial or complete absence of one X chromosome (45,X karyotype). Often, this pathology is diagnosed at birth due to heart problems, an unusually wide neck or swelling of the hands and feet. In our case such symptoms as a short and webbed neck, swelling on the back of the neck can be manifested. During echocardiography patent foramen ovale $(3 \mathrm{~mm})$ and bicuspid aortic valve have been revealed. However, it is also common for it to go undiagnosed for several years, often until the girl reaches the age of puberty and fails to develop typically (the changes associated with puberty do not occur). Despite the excellent postnatal prognosis, 99\% of Turner syndrome conceptions are thought to end in miscarriage or stillbirth,[2] and as many as $15 \%$ of all spontaneous abortions have the 45,X karyotype [3].

Cardiovascular abnormalities are common in TS. Besides, they constitute an important cause of early mortality [4]. The rate of cardiovascular malformations among patients with Turner syndrome ranges from $17 \%$ [5] to $45 \%$ [6]. Surveillance for aortic root dilatation, treatment for hypertension and prophylactic medical therapy with timely surgical consultation are essential to reduce the incidence of aortic dissection (risk is 1 in 40 per 100,000 TS person years). It also appears to occur several decades earlier than in the general female population [7]. Up to $15 \%$ of adults with Turner syndrome have bicuspid aortic valves, meaning only two, instead of three, parts to the valves in the main blood vessel leading from the heart are present. Since bicuspid valves are capable of regulating blood flow properly, this condition may go undetected without regular screening. However, bicuspid valves are more likely to deteriorate and later fail. Calcification also occurs in the valves, which may lead to a progressive valvular dysfunction as evidenced by aortic stenosis or regurgitation [8]. Therefore, it is important to conduct echocardiography, ECG and other examinations to identify these defects and henceforth correct them for a favorable prognosis in newborns in the future.

The causes of neonatal jaundice in this case were hemolysis of erythrocytes and maybe fetal infections (mother have ARVI 3 times during pregnancy). Jaundice occurred on the first day after birth. Neonatal jaundice and heart defects are associated with congenital hypothyroidism. In order to exclude this disease, measurements of thyroid gland hormone levels (FT4) and thyroid: stimulating hormone (TSH) were held. The hormone levels were 
stable. To rule out other hereditary diseases, phenylalanine and 17: hydroxyprogesterone measurements were undertaken. No changes were detected. Consultation of geneticianand karyotyping was performed and Turner syndrome was diagnosed.

Screening programs for Turner syndrome include a wide range of examinations that allow to identify the disease and get a proper diagnosis. Prenatally, ultrasound findings of increased nuchal translucency, cystic hygroma, left sided obstructive cardiac anomalies (especially coarctation of the aorta) in the fetus are highly suggestive of TS [7]. Postnatally, clinical features, geneticians consultation and karyotype test allow to diagnose TS. Karyotyping may be undertaken in the presence of a single clinical feature such as fetal hydrops or cystic hygroma, unexplained short stature, delayed puberty, obstructive left: sided cardiac abnormality (such as a bicuspid aortic valve, coarctation, aortic stenosis, hypoplastic left heart syndrome or mitral valve abnormalities), characteristic facial features (such as a short broad neck with webbing, narrow palate, micrognathia, low set ears and down: slanted palpebral fissures with epicanthal folds), or in a couple presenting with infertility [7]. Karyotyping should also be undertaken if two or more features commonly associated with TS such as renal anomaly (hypoplasia, aplasia or horseshoe kidney), other cardiac anomalies (e.g. partial anomalous pulmonary venous return, atrial or ventricular septal defects), Madelung deformity, dysplastic nails, multiple nevi, neuropsychological issues, and hearing loss associated with short stature are seen in a girl [7]. TS diagnosis is based on clinical features (such as a wide or weblike neck, low: set ears, broad chest with widely spaced nipples, swelling of the hands and feet, especially at birth, slightly smaller than average height at birth, slowed growth, cardiac defects, low hairline at the back of the head etc.) in countries with no screening programs.

The treatment of Turner syndrome is directed toward the specific symptoms that are apparent in each individual. In this case, application of phototherapy allowed to reduce symptoms of neonatal jaundice and to stabilize the newborn's condition. Vitamins and feeding mixtures are provided for adequate nutrition in a newborn. The child was discharged from the hospital in good conditions. The key aspects of managing Turner syndrome in children are cardiovascular monitoring and treatment of congenital heart disease; growth hormone therapy to augment linear growth (as early as 12 to 24 months of age); and supplemental estrogen therapy for sexual development and preservation of bone mineral density (typically initiated in the preteen years) [1]. Treatment may require the coordinated efforts of a team of specialists: pediatricians, surgeons, cardiologists, endocrinologists, speech pathologists, otolaryngologists, ophthalmologists, psychologists, and other healthcare professionals may need to systematically and comprehensively plan an effective child's treatment. Genetic counseling is recommended for affected individuals and their families.

\section{Conclusion}

Early diagnosis and proper treatment of Turner syndrome prevent the development of multiple complications that can appear in future. Moreover, they ensure leading healthy, productive, fulfilling life.

\section{References}

1. Morgan T (2007) Turner syndrome: Diagnosis and management. Am Fam Physician 76(3): 405-417.

2. Danielsson K (2009) Turner syndrome (monosomy X) and pregnancy loss.

3. Curtis M, Antoniewicz L, Linares ST (2014) Glass' office gynecology. Lippincott Williams \& Wilkins, USA, pp. 226.

4. Schoemaker MJ, Swerdlow AJ, Higgins CD, Wright AF, Jacobs PA, et al. (2008) Mortality in women with turner syndrome in great Britain: A national cohort study. J Clin Endocrinol Metab 93: 4735-4742.

5. Saenger P, Wikland KA, Conway GS, Davenport M, Gravholt $\mathrm{CH}$, et al. (2001) Recommendations for the diagnosis and management of turner syndrome. J Clin Endocrinol Metab 86(7): 3061-3069.

6. Dawson-Falk KL, Wright AM, Bakker B, Pitlick PT, Wilson DM, et al. (1992) Cardiovascular evaluation in turner syndrome: Utility of MR imaging. Australas Radiol 36(3): 204-209.

7. Shankar RK, Backeljauw PF (2018) Current best practice in the management of turner syndrome. Ther Adv Endocrinol Metab 9(1): 3340.

8. Elsheikh M, Dunger DB, Conway GS, Wass JA (2002) Turner's syndrome in adulthood. Endocr Rev 23(1): 120-140. 